Shipan Lu, Teachers College, Jimei University, Xiamen 361021, P. R. China. e-mail: libtc@jmu.edu

\title{
THE HAUSDORFF DIMENSION AND MEASURE OF SOME CANTOR SETS
}

\begin{abstract}
It will be shown that the density formula of [3] is proved by new method. As an application, the Hausdorff dimensions of and the Hausdorff measures of some Cantor-type sets will be evaluated.
\end{abstract}

\section{Basic concepts}

In this paper we denote by $M$ a compact interval on the real line, and we always assume the sets involved to be in $M$.

The following concepts and their related properties in this section can be found in [1] [2] [3].

Definition 1.1. (a) A non-negative function of sets $\mu$ is called a measure on $M$ if (i) $\mu(\phi)=0$, and (ii) $\mu(E) \leq \sum_{k=1}^{\infty} \mu\left(E_{k}\right)$, whenever $E \subseteq \cup_{k=1}^{\infty} E_{k}$. Here we don't draw a clear distinction between measure and outer measure as in [2] and [3].

(b) $A$ set $A$ is $\mu$-measurable if for each set $E, \mu(E)=\mu(E \cap A)+\mu(E \backslash A)$.

(c) $A$ measure $\mu$ is regular if for each set $E$ there exists a $\mu$-measurable set $A$ such that $E \subseteq A$ and $\mu(E)=\mu(A)$.

(d) A measure $\mu$ is Borel regular if every Borel set is $\mu$-measurable and for each set $E$ there exists a Borel set $A$ such that $E \subseteq A$ and $\mu(E)=\mu(A)$.

(e) A measure $\mu$ is a Radon measure if $\mu$ is Borel regular and $\mu(P)<\infty$ for each compact set $P$.

Definition 1.2. Let $E$ be a set. A sequence of closed intervals $\left\{I_{i}\right\}$ is called a $\delta$-cover of $E$ if $E \subset \cup_{i} I_{i}$ and $0<\left|I_{i}\right| \leq \delta$ for each $i$, where $|I|$ is the length of interval $I$. The s-dimensional Hausdorff measure of $E$ is defined by

$$
\mathcal{H}^{s}(E)=\lim _{\delta \rightarrow 0} \mathcal{H}^{s}(E)=\lim _{\delta \rightarrow 0} \inf \sum_{i}\left|I_{i}\right|^{s},
$$

Key Words: Hausdorff dimension, Hausdorff measure, Density formula

Mathematical Reviews subject classification: 28A80, 26A39

Received by the editors June 2, 1998

*The work on this research has been supported by JUTCSF. 
where the infimum is taken over all $\delta$-covers $\left\{I_{i}\right\}$ of $E$.

The Hausdorff dimension of $E$ is defined by

$$
\operatorname{dim}_{H} E=\sup \left\{s>0: \mathcal{H}^{s}(E)=\infty\right\}\left(=\inf \left\{s>0: \mathcal{H}^{s}(E)=0\right\}\right) .
$$

It is not difficult to verify that $\mathcal{H}^{s}$ is a regular metric measure, so is a regular Borel measure.

Definition 1.3. A collection of sets $\mathcal{M}$ is called a Vitali class of $E$ if for each $x \in E$ and $\delta>0$ there exists $U \in \mathcal{M}$ with $x \in U$ and $0<|U| \leq \delta$ where $|U|$ is the diameter of $U$.

Theorem 1.4 (Vitali covering theorem). Let $\mathcal{M}=\{I\}$ be a Vitali class of closed intervals of $E$. Then we may select a (finite or countable) nonoverlapping sequence $\left\{I_{i}\right\}$ from $\mathcal{M}$ such that either $\sum_{i}\left|I_{i}\right|^{s}=\infty$ or $\mathcal{H}^{s}\left(E \backslash \cup_{i}\right.$ $\left.I_{i}\right)=0$.

\section{The Density Formula}

In this section the symbol $\mu$ is always assumed to be a finite Radon measure and $E$ a Borel subset of $M$. The lower inverse s-density of $\mu$ at $x$ is defined by

$$
\underline{D}_{\mu} \mathcal{H}^{s}(x)=\lim _{\delta \rightarrow 0} \inf \frac{|I|^{s}}{\mu(I)}
$$

where the infimum is taken over all closed intervals $I$ with $x \in I$ and $|I|<\delta$. When $\mu(I)=0$, define $\underline{D}_{\mu} \mathcal{H}^{s}(x)=\infty$. We can show that the function $\underline{D}_{\mu} \mathcal{H}^{s}$ is a Borel function.

Lemma 2.1. If $\underline{D}_{\mu} \mathcal{H}^{s}(x) \geq c>0$ for each $x \in E$, then $\mathcal{H}^{s}(E) \geq c \mu(E)$. Thus $\operatorname{dim}_{H} E \geq s$.

Proof. Let $c^{*}>0$ with $c^{*}<c$. Since $\underline{D}_{\mu} \mathcal{H}^{s}(x)>c^{*}$ for each $x \in E$, there exists a positive function $\delta(x)$ on $E$ such that $\frac{|I|^{s}}{\mu(I)}>c^{*}$ for any $I$ satisfying $x \in I$ and $|I| \leq \delta(x)$. Define, for $n=1,2, \ldots, E_{n}=\left\{x \in E: \delta(x) \geq \frac{1}{n}\right\}$, we have $E_{n} \subseteq E_{n+1}, n=1,2, \ldots$, and $E=\cup_{n} E_{n}$. Let $\varepsilon>0$ and fix $n$. Take a $\frac{1}{n}$-cover $\left\{I_{i}\right\}$ of $E_{n}$ such that $\mathcal{H}^{s}\left(E_{n}\right) \geq \sum_{i}\left|I_{i}\right|^{s}+\varepsilon$, so

$$
\mathcal{H}^{s}\left(E_{n}\right)-\varepsilon \geq \sum_{i}\left|I_{i}\right|^{s}>c^{*} \sum_{i} \mu\left(I_{i}\right) \geq c^{*} \mu\left(E_{n}\right) .
$$

Making $\varepsilon \rightarrow 0$, we have

$$
\mathcal{H}^{s}(E) \geq \mathcal{H}^{s}\left(E_{n}\right) \geq c^{*} \mu\left(E_{n}\right)
$$


for all $n$. Since $\mu$ is a regular measure, we have

$$
\mathcal{H}^{s}(E) \geq c^{*} \lim _{n \rightarrow \infty} \mu\left(E_{n}\right)=c^{*} \mu(E) .
$$

Let $c^{*} \rightarrow c$, then the Lemma follows.

Lemma 2.2. If $\underline{D}_{\mu} \mathcal{H}^{s}(x) \leq c$ with $c>0$ for each $x \in E$, then $\mathcal{H}^{s}(E) \leq$ $c \mu(E)$. Thus, $\operatorname{dim}_{H} E \leq s$.

Proof. Let $\varepsilon>0$ and $c^{*}>c$ be given. Since $\mu$ is a Radon measure, there exists an open set $G$ such that $E \subseteq G$ and $\mu(G)<\mu(E)+\varepsilon$. Let $n$ be a positive integer and let

$$
\mathcal{M}_{n}=\left\{I: I \subseteq G,|I| \leq \frac{1}{n} \text { and }|I|^{s}<c \mu(I)\right\} .
$$

Since $\underline{D}_{\mu} \mathcal{H}^{s}(x)<c^{*}$ for each $x \in E, \mathcal{M}_{n}$ is a Vitali class of $E$. By the Vitali covering theorem, there is a non-overlapping subsequence $\left\{I_{i}^{n}\right\}$ of $\mathcal{M}_{n}$ such that

$$
\mathcal{H}^{s}\left(E \backslash \cup_{i} I_{i}^{n}\right)=0
$$

because of $\sum_{i}\left|I_{i}^{n}\right|^{s}<\sum_{i} c^{*} \mu\left(I_{i}^{n}\right)<c^{*} \mu(M)<\infty$. Let

$$
Z=\cup_{n}\left(E \backslash \cup_{i} I_{i}^{n}\right)
$$

Then $\mathcal{H}^{s}(Z)=0$ and $\mathcal{H}^{s}(E \backslash Z)=\mathcal{H}^{s}(E)$. By the fact that

$$
E \backslash Z=\cap_{n}\left(E \backslash\left(E \backslash \cup_{i} I_{i}^{n}\right)\right) \subset \cap_{n}\left(\cup_{i} I_{i}^{n}\right),
$$

we have

$$
\begin{aligned}
\mathcal{H}^{s}(E) & =\mathcal{H}^{s}(E \backslash Z) \leq \lim _{n \rightarrow \infty} \mathcal{H}_{\frac{1}{n}}^{s}\left(\cup_{i} I_{i}^{n}\right) \\
& \leq \lim _{n \rightarrow \infty} \sum_{i}\left|I_{i}^{n}\right|^{s} \leq \lim _{n \rightarrow \infty} c^{*} \sum_{i} \mu\left(I_{i}^{n}\right) \\
& \leq c^{*} \mu(G) \leq c^{*}(\mu(E)+\varepsilon) .
\end{aligned}
$$

Let $\varepsilon \rightarrow 0$ and $c^{*} \rightarrow c$,it follows that

$$
\mathcal{H}^{s}(E) \leq c \mu(E)
$$

Corollary. Let $Z=\left\{x \in E: \underline{D}_{\mu} \mathcal{H}^{s}(x)=0\right\}$, then $\mathcal{H}^{s}(Z)=0$.

Proof. Write $E_{i}=\left\{x \in E: \underline{D}_{\mu} \mathcal{H}^{s}(x)<i^{-1}\right\}$. Then $Z \subseteq \cap_{i=1}^{\infty} E_{i}$. For each $i$, by Lemma 2.2, we have

$$
\mathcal{H}^{s}\left(E_{i}\right) \leq i^{-1} \mu\left(E_{i}\right) \leq i^{-1} \mu(M),
$$

and this gives $\mathcal{H}^{s}(Z)=0$. 
Theorem 2.3. If $\underline{D}_{\mu} \mathcal{H}^{s}(x)<\infty$ for each $x \in E$, then

$$
\mathcal{H}^{s}(E)=\int_{E} \underline{D}_{\mu} \mathcal{H}^{s} d \mu
$$

Proof. Let $Z=\left\{x \in E: \underline{D}_{\mu} \mathcal{H}^{s}(x)=0\right\}$. Then $\mathcal{H}^{s}(Z)=0$ by the Corollary of Lemma 2.2, and $\mathcal{H}^{s}(E)=\mathcal{H}^{s}\left(E_{+}\right)$, where $E_{+}=\left\{x \in E: \underline{D}_{\mu} \mathcal{H}^{s}(x)>0\right\}$. Fix $1<t<\infty$. Let $E_{m}=\left\{x \in E: t^{m} \leq \underline{D}_{\mu} \mathcal{H}^{s}(x)<t^{m+1}\right\}$, then $E_{+}=$ $\cup_{m=-\infty}^{\infty} E_{m}$. Since

$$
\begin{aligned}
\mathcal{H}^{s}\left(E_{+}\right) & =\sum_{m} \mathcal{H}^{s}\left(E_{m}\right) \leq \sum_{m} t^{m+1} \mu\left(E_{m}\right) \quad \text { (by Lemma 2.2) } \\
& =t \sum_{m} t^{m} \mu\left(E_{m}\right) \leq t \sum_{m} \int_{E_{m}} \underline{D}_{\mu} \mathcal{H}^{s} d \mu=t \int_{E_{+}} \underline{D}_{\mu} \mathcal{H}^{s} d \mu
\end{aligned}
$$

and

$$
\begin{aligned}
\mathcal{H}^{s}\left(E_{+}\right) & =\sum_{m} \mathcal{H}^{s}\left(E_{m}\right) \geq \sum_{m} t^{m} \mu\left(E_{m}\right) \quad \text { (by Lemma 2.1) } \\
& =\frac{1}{t} \sum_{m} t^{m+1} \mu\left(E_{m}\right) \geq \frac{1}{t} \sum_{m} \int_{E_{m}} \underline{D}_{\mu} \mathcal{H}^{s} d \mu=\frac{1}{t} \int_{E_{+}} \underline{D}_{\mu} \mathcal{H}^{s} d \mu,
\end{aligned}
$$

then by sending $t \rightarrow 1^{+}$, we have

$$
\mathcal{H}^{s}(E)=\mathcal{H}^{s}\left(E_{+}\right)=\int_{E_{+}} \underline{D}_{\mu} \mathcal{H}^{s} d \mu=\int_{E} \underline{D}_{\mu} \mathcal{H}^{s} d \mu .
$$

\section{Some Cantor Type Sets}

(1) A Simple Cantor set. Let $0<s \leq 1, n_{k}$ be a sequence of positive integers with $n_{k} \geq 2$ for each $k,\left\{c_{k}\right\}$ be a sequence of positive real numbers with $n_{k} c_{k}^{s}=1$ for each $k$. Let $E_{0}=I^{(0)}$ be the real interval [0,1]. Suppose $E_{k}$ has been defined and consists of $i_{k}$ equal closed intervals $I_{i}^{(k)}, i=1,2, \ldots, i_{k}$. Then $E_{k+1}$ is obtained from $E_{k}$ by removing, from each $I_{i}^{(k)},\left(n_{k+1}-1\right)$ equal open intervals $G_{i}^{(k+1)}$ leaving $n_{k+1}$ closed intervals each of which with length $c_{k+1}\left|I_{i}^{(k)}\right|$. Let

$$
E=\bigcap_{k=0}^{\infty} E_{k}=\bigcap_{k=0}^{\infty} \bigcup_{i=1}^{i_{k}} I_{i}^{(k)},
$$


it is called a simple Cantor set. We shall show that $\operatorname{dim}_{H} E=s$ and $\mathcal{H}^{s}(E)=$ 1.

These intervals $I_{i}^{(k)}, i=1,2, \ldots, i_{k}$, are called basic intervals of $\mathrm{k}^{\prime} t h$ stage which are generally written by $I^{(k)}$ since their length are equal, and we have $i_{k}=n_{1} n_{2} \ldots n_{k}$ by the construction. Similarly, all $G_{i}^{(k)}, i=1,2, \ldots, i_{k}-$ $i_{k-1}$, are called the gap of $k^{\prime}$ th stage which are generally written by $G^{(k)}$ since their length are equal. Now define a function $\mu$ of sets on $[0,1]$ by $\mu\left(I^{(k)}\right)=\left|I^{(k)}\right|^{s}$. Then we extend $\mu$ to a mass distribution on $[0,1]$ whose support is $E$ since $\mu\left(I^{(k)}\right)=\left|\frac{I^{(k+1)}}{c_{k+1}}\right|^{s}=c_{k+1}^{-s}\left|I^{(k+1)}\right|^{s}=n_{k+1} \mu\left(I^{(k+1)}\right)$ (cf. Proposition 1.7 of [2]). Clearly $\mu$ is a Radon measure and $\mu(E)=|[0,1]|^{s}=1$. In the following we shall show $\underline{D}_{\mu} \mathcal{H}^{s}(x)=1$ for each $x \in E$, then by the Theorem 2.3 we have

$$
\mathcal{H}^{s}(E)=\int_{[0,1]} \underline{D}_{\mu} \mathcal{H}^{s} d \mu=\mu([0,1])=1
$$

and $\operatorname{dim}_{H} E=s$.

Firstly, for each $x \in E$ there is a sequence $\left\{I_{j_{k}}^{(k)}\right\}$ of basic intervals such that $x \in \bigcap_{k=1}^{\infty} I_{j_{k}}^{(k)}$. Since $\left|I_{j_{k}}^{(k)}\right| \rightarrow 0(k \rightarrow \infty)$ and $\left|I_{j_{k}}^{(k)}\right|^{s}=\mu\left(I_{j_{k}}^{(k)}\right)$ we have $\underline{D}_{\mu} \mathcal{H}^{s}(x) \leq 1$ for $x \in E$.

Secondly, in order to prove $\underline{D}_{\mu} \mathcal{H}^{s}(x) \geq 1$, we have to show that $|I|^{s} \geq \mu(I)$ for any interval $I$ which contains $x \in E$. We draw up two steps to this end.

(i) A closed interval $I$ is called a near basic interval if its lift end point coincides with the lift end points of some basic intervals and its right end point coincides with the right end points of some basic intervals. For a near basic interval, we always combine the basic intervals into bigger (of lower stage) basic intervals if possible, so the basic intervals which contained in a near basic interval are finite and possessed of definite type. Now we should prove that $|I|^{s} \geq \mu(I)$ for any near basic intervals by using the induction for the number of basic intervals in $I$. When $I$ contains only one basic interval, $I$ itself is the basic interval, so $|I|^{s}=\mu(I)$. Now suppose that $I$ contains $n$ basic intervals, and is contained in a basic interval of $(k-1)$ 'th stage rather than in a basic interval of $k^{\prime}$ th stage. Then $I$ is in one of the three cases: (a) $I$ contains exactly $n$ basic intervals of $k^{\prime}$ th stage, so $|I|=n\left|I^{(k)}\right|+(n-1)\left|G^{(k)}\right|$, $n \leq n_{k}$; (b) $I=I_{1} \cup I_{2}$, where $\left|I_{2}\right|=m\left|I^{(k)}\right|+m\left|G^{(k)}\right|, m<n_{k}$, and $I_{1}$, which is contained in a basic interval of $k$ 'th stage, is a near basic interval on one of the side of $I_{2} ;\left(\right.$ c) $I=I_{1} \cup I_{2} \cup I_{3}$, where $\left|I_{2}\right|=m\left|I^{(k)}\right|+(m+1)\left|G^{(k)}\right|$, $m<n_{k}-1$, and $I_{1}$ and $I_{3}$, which are contained in some basic intervals of $k$ 'th stage, are the near basic intervals on two sides of $I_{2}$ respectively. For the case (a), we consider the function $f(x)=\left(x\left|I^{(k)}\right|+(x-1)\left|G^{(k)}\right|\right)^{s}-x\left|I^{(k)}\right|^{s}$. Since $f^{\prime \prime}(x)<0, x \in\left[1, n_{k}\right], f(x)$ is convex. Again since $f(1)=f\left(n_{k}\right)=0$, we 
have $f(x) \geq 0, x \in\left[1, n_{k}\right]$, that is $|I|^{s} \geq n\left|I^{(k)}\right|^{s}=\mu(I)$. For the case (b), we consider the function $g(x)=\left(m\left|I^{(k)}\right|+m\left|G^{(k)}\right|+x\left|I^{(k)}\right|\right)^{s}-m\left|I^{(k)}\right|^{s}-\left(x\left|I^{(k)}\right|\right)^{s}$. By (a), we have $g(0)>0, g(1) \geq 0$. And since $g^{\prime}(x)<0, x \in(0,1], g(x) \geq 0$, $x \in[0,1]$, we have $|I|^{s} \geq m\left|I^{(k)}\right|^{s}+\left|I_{1}\right|^{s}$. We have inductively $\left|I_{1}\right|^{s} \geq \mu\left(I_{1}\right)$, so $|I|^{s} \geq \mu\left(I_{1}\right)+\mu\left(I_{2}\right)=\mu(I)$. For the case (c), we consider the function $h(x, y)=$ $\left(m\left|I^{(k)}\right|+(m+1)\left|G^{(k)}\right|+x\left|I^{(k)}\right|+y\left|I^{(k)}\right|\right)^{s}-m\left|I^{(k)}\right|^{s}-\left(x\left|I^{(k)}\right|\right)^{s}-\left(y\left|I^{(k)}\right|\right)^{s}$. By (b), we have $h(x, 0)>0, h(x, 1) \geq 0, x \in[0,1]$. And since $h_{y}^{\prime}(x, y)<0,0 \leq$ $x, y \leq 1, h(x, y) \geq 0,0 \leq x, y \leq 1$, we have $|I|^{s} \geq m\left|I^{(k)}\right|^{s}+\left|I_{1}\right|^{s}+\left|I_{3}\right|^{s}$. We have inductively $\left|I_{1}\right|^{s} \geq \mu\left(I_{1}\right),\left|I_{3}\right|^{s} \geq \mu\left(I_{3}\right)$, so $|I|^{s} \geq \mu\left(I_{1}\right)+\mu\left(I_{2}\right)+\mu\left(I_{3}\right)=$ $\mu(I)$.

(ii) Now for any $x \in E$ and any interval $I$ with $x \in I$, we can assume that the two end points of $I$ belong to E, otherwise we will contract $I$ without decrease $\frac{|I|^{s}}{\mu(I)}$. For this $I$, we take a sequence $\left\{I_{k}\right\}$ of near basic intervals such that $I_{k} \subset I$ and $\lim _{k \rightarrow \infty} I_{k}=I$. By the continuity of the power function $x^{s}$ and the measure $\mu$, we have $\frac{|I|^{s}}{\mu(I)}=\lim _{k \rightarrow \infty} \frac{\left|I_{k}\right|^{s}}{\mu\left(I_{k}\right)}$. Since $\left|I_{k}\right|^{s} \geq \mu\left(I_{k}\right)$ for each $\mathrm{k},|I|^{s} \geq \mu(I)$. Thus $\underline{D}_{\mu} \mathcal{H}^{s}(x) \geq 1$.

Now we have proved $\underline{D}_{\mu} \mathcal{H}^{s}(x)=1$ for any $x \in E$ and the conclusion follows.

(2) A homogeneous Cantor set. Let $\left\{n_{k}\right\}$ be a sequence of positive integers with $n_{k} \geq 2$ for each $k,\left\{c_{k}\right\}$ be a sequence of positive numbers with $n_{k} c_{k} \leq 1$ for each $k$. Let $E_{0}=I^{(0)}$ be the real interval $[0,1]$. Suppose that $E_{k}$ has been defined and consists of $i_{k}$ equal closed intervals $I_{i}^{(k)}, i=1,2, \ldots, i_{k}$. We can obtain $E_{k+1}$ from $E_{k}$ by removing, from each $I_{i}^{(k)},\left(n_{k+1}-1\right)$ equal open intervals $G_{j}^{(k+1)}$ leaving $n_{k+1}$ closed intervals each of which with length $c_{k+1}\left|I_{i}^{(k)}\right|$. Let

$$
E=\bigcap_{k=0}^{\infty} E_{k}=\bigcap_{k=0}^{\infty} \bigcup_{i=1}^{i_{k}} I_{i}^{(k)}
$$

it is called a homogeneous Cantor set.

As same as in the simple Cantor set, for each positive integer $k, i_{k}=$ $n_{1} n_{2} \ldots n_{k}$; all basic intervals $I_{i}^{(k)}$ of $k$ 'th stage, $i=1,2, \ldots, i_{k}$, each of which has equal length, are written by $I^{(k)}$; all gap $G_{i}^{(k)}$ of $k^{\prime}$ 'th stage, $i=$ $1,2, \ldots, i_{k}-i_{k-1}$, each of which has equal length, are written by $G^{(k)}$. Let $s_{k}=\frac{\log \left(n_{1} n_{2} \ldots n_{k}\right)}{-\log \left(c_{1} c_{2} \ldots c_{k}\right)}, \underline{s}=\lim _{k \rightarrow \infty} \inf s_{k}$, we shall show that $\operatorname{dim}_{H} E=\underline{s}$.

We define a function $\mu$ of sets on $[0,1]$ by $\mu\left(I^{(0)}\right)=1, \mu\left(I^{(k)}\right)=$ $\left(n_{1} n_{2} \ldots n_{k}\right)^{-1}, k=1,2, \ldots$, and then extend $\mu$ to a mass distribution on $[0,1]$ whose support is $E$ since $\mu\left(I^{(k)}\right)=n_{k+1} \mu\left(I^{(k+1)}\right)$. By taking notice of $\left|I^{(k)}\right|=c_{1} c_{2} \ldots c_{k}$ and $n_{1} n_{2} \ldots n_{k}\left(c_{1} c_{2} \ldots c_{k}\right)^{s_{k}}=1$, we have $\mu\left(I^{(k)}\right)=$ 
$\left|I^{(k)}\right|^{s_{k}}$.

Let $t>\underline{s}$. Then there is a sequence $\left\{j_{k}\right\}$ of natural numbers such that $s_{j_{k}}<t$ for each $k$. For any $x \in E$ there is a sequence $\left\{I_{n\left(j_{k}\right)}^{\left(j_{k}\right)}\right\}$ of basic intervals such that $x \in \cap_{k=1}^{\infty} I_{n\left(j_{k}\right)}^{\left(j_{k}\right)}$, where $n\left(j_{k}\right) \in\left\{1,2, \ldots, i_{j_{k}}\right\}$. By

$$
\underline{D}_{\mu} \mathcal{H}^{t}(x) \leq \lim _{k \rightarrow \infty} \frac{\left|I^{\left(j_{k}\right)}\right|^{t}}{\mu\left(I^{\left(j_{k}\right)}\right)} \leq \lim _{k \rightarrow \infty} \frac{\left|I^{\left(j_{k}\right)}\right|^{s_{j_{k}}}}{\mu\left(I^{\left(j_{k}\right)}\right)}=1
$$

and Lemma 2.2, we have $\operatorname{dim}_{H} E \leq t$. Making $t \rightarrow \underline{s}$, we have $\operatorname{dim}_{H} E \leq \underline{s}$.

In order to prove $\operatorname{dim}_{H} E \geq \underline{s}$, we only need to prove $\operatorname{dim}_{H} E \geq s$ for any $s \leq \underline{s}$. We define the near basic interval as in (1), and by Lemma 2.2 we need only to prove $|I|^{s} \geq d \mu(I)$ for any sufficiently small near basic intervals $I$, where $d$ is a constant. Since $s<\underline{s}$, there is a natural number $N$ such that $s_{k}>s$ for any $k \geq N$. Let $I$ be a sufficiently small near basic interval which is contained in a basic interval of $(k-1)^{\prime}$ th stage rather than in a basic interval of $k$ 'th stage, where $k>N$. Then $I=I_{1} \cup G^{(k)} \cup I_{2}$, where $I_{1}$ and $I_{2}$ are near basic intervals on two sides of $G^{(k)}$. Let the lowest stage of the basic intervals contained in $I_{1}$ be of $p$ 'th stage. Then $p \geq k$, so we can suppose that $I_{1}$ contains $m p$ 'th stage basic intervals and a near basic interval contained in some $I^{(p)}$. Write $I^{*}$, the near basic interval which is contained in $I_{1}$ and has $m$ basic intervals of $p$ th stage. Considering function $f(x)=\left(x\left|I^{(p)}\right|+(x-1)\left|G^{(p)}\right|\right)^{s}-x\left|I^{(p)}\right|^{s_{p}}$ and noticing $s<\min \left\{s_{p}, s_{p-1}\right\}$, we can prove $f(x) \geq 0, x \in\left[1, n_{p}\right]$, by the same method as in (1). Therefore we have $\left|I^{*}\right|^{s} \geq m\left|I^{(p)}\right|^{s_{p}}=m \mu\left(I^{(p)}\right)=\mu\left(I^{*}\right)$, consequently $\left|I_{1}\right|^{s} \geq\left|I^{*}\right|^{s} \geq$ $\frac{1}{2}(m+1) \mu\left(I^{(p)}\right) \geq \frac{1}{2} \mu\left(I_{1}\right)$. For the same reason we have $\left|I_{2}\right|^{s} \geq \frac{1}{2} \mu\left(I_{2}\right)$, and then $|I|^{s} \geq \frac{1}{2}\left(\left|I_{1}\right|^{s}+\left|I_{2}\right|^{s}\right) \geq \frac{1}{4}\left(\mu\left(I_{1}\right)+\mu\left(I_{2}\right)\right)=\frac{1}{4} \mu(I)$.

(3) A perturbed Cantor set. Let $\left\{c_{k j}\right\}_{k}(j=1,2)$ be two sequences of positive numbers, satisfying $c_{k 1}+c_{k 2}<1$ for each $k$ and $\lim _{k \rightarrow \infty} \inf c_{k j}>0$ for $j=1,2$. We construct, inductively, a sequence $\left\{E_{k}\right\}$ of sets : let $E_{0}=I_{\phi}=$ $[0,1]$; suppose $E_{k}=\cup_{\sigma \in\{1,2\}^{k}} I_{\sigma}$ has been defined, where $I_{\sigma}$ is a closed interval and $\sigma \in\{1,2\}^{k}$ is a mapping $\sigma:\{1,2, \ldots, k\} \rightarrow\{1,2\}$; for each $I_{\sigma}$, which is called a basic interval of $k$ 'th stage, we obtain $I_{\sigma, j}$ by removing an open interval $G_{\sigma}$ from $I_{\sigma}$ such that $\frac{\left|I_{\sigma, j}\right|}{\left|I_{\sigma}\right|}=c_{k+1, j}$, where $(\sigma, j) \in\{1,2\}^{k+1}$ such that $(\sigma, j)(t)=\sigma(t)$ whenever $t \in\{1,2, \ldots, k\}$ and $(\sigma, j)(k+1)=j, j=1,2$, then $E_{k+1}=\cup_{\sigma \in\{1,2\}^{k+1}} I_{\sigma}$. Let

$$
E=\bigcap_{k=0}^{\infty} E_{k}
$$

it is called a perturbed Cautor set. Let $s_{k}$ be a real number satisfying $\prod_{i=1}^{k}\left(c_{i 1}^{s_{k}}+c_{i 2}^{s_{k}}\right)=1$ and let $\underline{s}=\lim _{k \rightarrow \infty} \inf s_{k}$. We shall prove $\operatorname{dim}_{H} E=\underline{s}$. 
Let $s \in(0,1]$. Define a function $\mu$ of sets on $[0,1]$ such that $\mu\left(I_{\phi}\right)=1$ and

$$
\mu\left(I_{\sigma}\right)=\frac{\left|I_{\sigma}\right|^{s}}{\prod_{i=1}^{k}\left(c_{i 1}^{s}+c_{i 2}^{s}\right)}
$$

for each $\sigma \in\{1,2\}^{k}, k=1,2, \ldots$, then extend $\mu$ to a mass distribution on $[0,1]$ whose support is $E$ because of

$$
\begin{aligned}
& \sum_{j=1}^{2} \mu\left(I_{\sigma, j}\right)=\sum_{j=1}^{2} \frac{\left|I_{\sigma, j}\right|^{s}}{\prod_{i=1}^{k+1}\left(c_{i 1}^{s}+c_{i 2}^{s}\right)} \\
& =\frac{\left(c_{k+1,1}^{s}+c_{k+1,2}^{s}\right)\left|I_{\sigma}\right|^{s}}{\prod_{i=1}^{k+1}\left(c_{i 1}^{s}+c_{i 2}^{s}\right)}=\frac{\left|I_{\sigma}\right|^{s}}{\prod_{i=1}^{k}\left(c_{i 1}^{s}+c_{i 2}^{s}\right)}=\mu\left(I_{\sigma}\right) .
\end{aligned}
$$

Let $s>\underline{s}, s$ is presented in $(*)$. Then there is a sequence $\left\{j_{k}\right\}$ of natural numbers such that $s_{j_{k}}<s$ for each $k$. For any $x \in E$ there is a sequence $\left\{I_{\sigma_{k}}: \sigma_{k} \in\{1,2\}^{j_{k}}\right\}$ of basic intervals such that $x \in \cap_{k=1}^{\infty} I_{\sigma_{k}}$. Since $\left|I_{\sigma_{k}}\right| \rightarrow$ $0(k \rightarrow \infty)$ and $\frac{\left|I_{\sigma_{k}}\right|^{s}}{\mu\left(I_{\sigma_{k}}\right)}=\prod_{i=1}^{i_{k}}\left(c_{i 1}^{s}+c_{i 2}^{s}\right)<\prod_{i=1}^{i_{k}}\left(c_{i 1}^{s_{k}}+c_{i 2}^{s_{k}}\right)=1$, we have $\underline{D}_{\mu} \mathcal{H}^{s}(x) \leq 1$. It follows from the lemma 2.2 that $\operatorname{dim}_{H} E \leq s$, and we have $\operatorname{dim}_{H} E \leq \underline{s}$ by making $s \rightarrow \underline{s}$, .

In order to prove $\operatorname{dim}_{H} E \geq \underline{s}$, we only need to prove $\operatorname{dim}_{H} E \geq s$ for any $s \leq \underline{s}$. As in $(2)$, we need only to prove $|I|^{s} \geq d \mu(I)$ for any sufficiently small near basic intervals (it is defined as in (1)), where $d$ is a constant. Since $\lim _{k \rightarrow \infty} \inf c_{k_{j}}>0, j=1,2$, there are a positive number $c$ and a natural number $N^{*}$ such that $c_{k_{j}} \geq c$ for each $k \geq N^{*}$ and $j=1,2$. Let $s<\underline{s}, s$ is presented in $(*)$. Then there is a natural number $N$ such that $s_{k}>s$ for any $k \geq N$, so we have $\frac{\left|I_{\sigma}\right|^{s}}{\mu\left(I_{\sigma}\right)}=\prod_{i=1}^{k}\left(c_{i 1}^{s}+c_{i 2}^{s}\right)>\prod_{i=1}^{k}\left(c_{i 1}^{s_{i_{k}}}+c_{i 2}^{s_{i_{k}}}\right)=1$ for any basic intervals of $k$ 'th stage $I_{\sigma}$, we may require $N \geq N^{*}$ if necessary. Let $I$, a near basic interval, be contained in a basic interval of $(k-1)$ 'th stage, rather than in a basic interval of $k$ 'th stage. Then $I=I_{1} \cup G_{\sigma} \cup I_{2}$, where $\sigma \in\{1,2\}^{k-1}, I_{1}$ and $I_{2}$ are near basic intervals contained in $I_{\sigma, 1}$ and $I_{\sigma, 2}$ respectively. Let the lowest stage of the basic intervals contained in $I_{2}$ be $p^{\prime}$ th stage. Then $p \geq k$, and $I_{2}=I_{\sigma^{*}, 1}$, or $I_{\sigma^{*}, 1} \cup G_{\sigma^{*}} \cup I^{*}$, where $\sigma^{*} \in\{1,2\}^{p-1}$ and $I^{*}$ is a near basic interval contained in $I_{\sigma^{*}, 2}$. By $\left|I_{2}\right|^{s} \geq\left|I_{\sigma^{*}, 1}\right|^{s} \geq d^{s}\left|I_{\sigma^{*}}\right|^{s}$ and $\mu\left(I_{2}\right) \leq \mu\left(I_{\sigma^{*}}\right)$, we have $\frac{\left|I_{2}\right|^{s}}{\mu\left(I_{2}\right)} \geq \frac{d^{s}\left|I_{\sigma^{*}}\right|^{s}}{\mu\left(I_{\sigma^{*}}\right)} \geq d^{s}$. Similarly, we can prove $\frac{\left|I_{1}\right|^{s}}{\mu\left(I_{1}\right)} \geq d^{s}$, therefore $\frac{|I|^{s}}{\mu(I)} \geq \frac{1}{2} d^{s}$, and the conclusion follows.

Remark 3.1. The papers [7] and [6] have discussed the homogeneous Cantor set and the perturbed Cantor set respectively, but the methods we handle here are more simple and direct. 
The Hausdorff Dimension and Measure of Some Cantor Sets 807

\section{References}

[1] K. J. Falconer, The geometry of fractal sets, Cambridge Univ. Press, 1985.

[2] K. J. Falconer, Fractal geometry, John Wiley and Sons, 1990.

[3] L. C. Evens and R. F. Gariepy, Measure theory and fine properties of function, CRC Press. Inc., 1992.

[4] B. S. Thomson, Derivates of interval function, Memoirs of the Amer. Math. Soc. 452, 1991.

[5] G. A. Edgar, Fine variation and fractal measures, Real Analysis Exchange 20, 1994/1995.

[6] I. S. Baek, Dimensions of the perturbed Cantor set, Real Analysis Exchange, 19, No.1, 1993/1994.

[7] D. J. Feng ... [et al], On the net-measure properties and applications of homogeneous Cantor set, Advances in Natural Science, Vol.6, No.6, 1996(in Chinese). 
